\title{
Espacios-interfaz para una educación post-digital. Estrategias, instrumentos y recursos para propuestas didácticas innovadoras.
}

Interface-spaces for a post-digital education. Strategies, instruments and resources for innovative didactic proposals.

\author{
> Maria Elena Tosello \\ Universidad Nacional del Litoral, Argentina \\ mtosello@fadu.unl.edu.ar
}

\author{
> Maria Georgina Bredanini Colombo \\ Universidad Nacional del Litoral, Argentina \\ gbredani@fadu.unl.edu.ar
}

\begin{abstract}
In the current sociotechnical scenario, university education must adapt imaginatively its objectives and methods, integrating educational networks in hybrid spaces, starting from a model oriented to contextual learning by a distributed system of building knowledge, stimulating the development of interpersonal skills and abilities for collaborative problem solving. The course "Educational Interfaces. Strategies, Instruments and Spaces for Innovative Didactic Proposals “, intended for professors of design disciplines, sought to motivate novel educational processes which integrate the potentialities of digital media, through blended learning methodologies that complement face-to-face classes with resources and activities in space-interfaces, to offer more opportunities of learning.
\end{abstract}

Keywords: Interface-Space; Design Education; Blended Learning; Moodle; Open Access

\section{Problemática}

La Universidad Nacional del Litoral, en su Plan de Desarrollo Institucional 2010-2019, plantea que la sociedad del conocimiento se caracteriza por su capacidad de producir, transformar, difundir y utilizar la información para crear y aplicar conocimientos al desarrollo humano y social, y que el avance científico-tecnológico y la apropiación social de los conocimientos, son aspectos esenciales en la configuración del mundo actual. Este escenario impacta profundamente en los procesos educativos, por lo cual es necesario sostener una permanente reflexión sobre el quehacer docente. Abrir espacios de innovación curricular implica que las propuestas pedagógicas y las prácticas educativas sean repensadas en el nuevo contexto, constituyéndose en instancias que posibiliten el desarrollo profesional docente -resignificando su función- y contribuyan a mejorar los procesos de enseńanza-aprendizaje (PDI, UNL, 2010).

Hoy el conocimiento se construye en red y crece exponencialmente, y los entornos virtuales interactivos de Acceso Abierto son apropiados por diversas comunidades para almacenar información, compartir conocimientos y desarrollar experiencias colectivas. En este contexto socio-técnico, la educación universitaria debe adaptar sus objetivos y métodos con imaginación, conformando comunidades de formación en red y espacios educativos híbridos, a partir de un modelo orientado al aprendizaje contextual por un sistema distribuido de construcción de conocimientos, que favorezca el desarrollo de destrezas interpersonales y competencias para la innovación y la resolución colaborativa de problemas, recreando la cultura de modo crítico y emancipador (Freire, 1989).
En un modelo didáctico innovador, no es suficiente utilizar nuevos instrumentos y metodologías proyectuales, si no se enmarcan en propuestas educativas que integren los estudios presenciales con las tecnologías del aprendizaje y el conocimiento-TAC-, para favorecer un acceso creciente y sostenido a una educación inclusiva (PDI, UNL, 2010).

\section{Propuesta}

El curso "Interfaces Educativas. Estrategias, Instrumentos y Espacios para Propuestas Didácticas Innovadoras", responde a la necesidad de fortalecimiento de la planta docente y de renovación de la propuesta curricular de la Facultad de Arquitectura, Diseño y Urbanismo de la UNL, en relación a las expectativas de su comunidad educativa. El curso constituyó un espacio de reflexión sobre las prácticas educativas para analizar cómo se enseñan y, fundamentalmente, cómo se aprenden las disciplinas proyectuales en la actualidad, a fin de motivar a los docentes a diseñar propuestas educativas flexibles y en relación al perfil de los estudiantes actuales (nativos digitales), buscando maneras creativas de promover aprendizajes profundos a partir de actividades conectadas con sus intereses (Grané Oró, 2013), y la participación en acciones colaborativas que favorecen la construcción de un pensamiento autónomo y crítico, apoyado en el compromiso y la habilidad del trabajo "entre" sujetos, objetos, espacios y medios (Bessone y Garramuño, 2009).

Durante el curso se analizaron alternativas para la integración efectiva de las potencialidades de las TAC, mediante metodologías de aprendizaje combinado que interrelacionan los procesos presenciales con recursos y actividades en entornos virtuales, las cuales fueron 

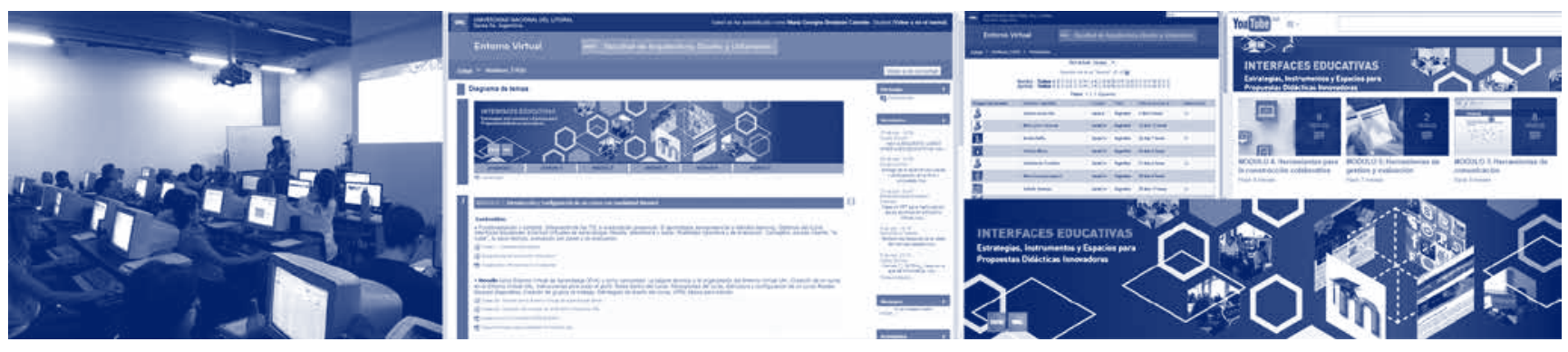

Figura 1: Clases presenciales y espacio-interfaz de interacción del curso.

experimentadas por los propios docentes en un espacio-interfaz de trabajo común que procuró el intercambio dialógico entre los profesores (Fig. 1). En paralelo, se realizaron prácticas sobre múltiples instrumentos y servicios online para incorporarlos en los procesos formativos, y se abrieron Aulas Virtuales en la plataforma de la universidad destinadas a las asignaturas de los docentes participantes.

El trabajo final consistió en elaborar una propuesta didáctica innovadora para la asignatura de pertenencia, orientada a propiciar aprendizajes significativos a partir de la construcción compartida de los conocimientos, repensando objetivos, contenidos, prácticas y criterios de evaluación. La propuesta debía incluir el diseño y desarrollo de los espacios-interfaz donde se experimentarían las nuevas experiencias; el diseño, producción e integración de recursos, servicios y actividades online; y un sistema de evaluación pertinente.

\section{Metodología}

El curso se desarrolló sobre la base de cinco módulos temáticos: Introducción y configuración del curso; Instrumentos para generar e incorporar recursos en la plataforma; Herramientas de comunicación; Herramientas para la construcción colaborativa de conocimientos; y Herramientas de gestión y evaluación, que se implementaron a través de 6 clases con una carga horaria de 30 horas presenciales; 15 horas de tutorías; más 15 horas de trabajo autónomo. Fue dictado por once profesores: responsables, colaboradores e invitados; y en condición de alumnos participaron 37 docentes: titulares, adjuntos y auxiliares de las carreras Arquitectura y Urbanismo, y Licenciaturas en Diseño de la Comunicación Visual y en Diseño Industrial.

La organización de cada encuentro combinó clases teóricas dictadas por los docentes a cargo del curso; clases prácticas dictadas por los docentes colaboradores; conferencias sobre las experiencias desarrolladas por los docentes invitados; y actividades de taller destinadas a la realización de trabajos prácticos parciales. Las clases de tutorías fueron destinadas a la orientación y seguimiento académico de las propuestas, y al finalizar, se realizó una jornada de presentación de los trabajos finales. Durante todo el curso se brindó apoyatura desde la plataforma educativa en el entorno Moodle.

\section{Perfil de los Cursantes}

Los docentes que realizaron el curso en calidad de alumnos son en su mayoría Auxiliares en Docencia (Jefes de Trabajos Prácticos y Ayudantes), algunos son pasantes-alumnos y graduados, y sólo unos pocos son docentes con cargos profesorales (titulares y adjuntos).
La franja etaria de los cursantes se ubica entre los 25 y los 55 años, lo que permitió formar equipos de trabajo integrando "nativos" e “inmigrantes" digitales (Piscitelli, 2009), para articular el cruce de saberes y habilidades diversas, y el afianzamiento de los aprendizajes.

A los efectos de definir qué experiencias y destrezas poseían los participantes en relación a la temática del curso, se realizó una encuesta. En primer lugar fueron consultados acerca del uso de Internet: más del $90 \%$ afirmó que utiliza Internet hace más de 10 años; que acceden a la web varias veces al día; y que permanecen conectados más de 6 horas diarias. Afirmaron utilizar la web para actividades académicas, profesionales, sociales y de comunicación. Sin embargo, al ser consultados sobre qué servicios de Internet utilizan regularmente, la mayoría respondió que utiliza el correo electrónico, los buscadores y redes sociales, y en menor medida, los sitios y servicios educativos, foros o blogs temáticos. Ante la pregunta de si consideran que los espacios de Internet forman parte de su hábitat, el 90\% ha respondido que sí. El 95\% de los docentes encuestados declaró disponer de un espacio personal en Internet, pero al solicitarles que indiquen de qué tipo de espacios se trata, el mismo porcentaje indicó poseer una página en Facebook, y en menor medida en Google+. Consultados sobre si antes de realizar el curso utilizaban algún medio de comunicación con los alumnos -fuera de las clases presenciales-, el 95\% respondió afirmativamente, entre ellos la mayoría utiliza el correo electrónico y/o Facebook.

En este contexto, uno de los grandes desafíos del curso fue estimular a los docentes -habituados de algún modo al uso de las TICa integrar estos instrumentos en propuestas didácticas innovadoras, transformándolas en verdaderas tecnologías del aprendizaje y el conocimiento. En este sentido, se buscó promover la renovación de las estrategias, espacios y recursos educativos, en relación a modalidades que se ajusten a las prácticas propias de las disciplinas proyectuales.

\section{Contenidos del Curso}

Se brindaron fundamentos teóricos sobre la integración de las TIC a la educación presencial, bajo la modalidad de aprendizaje combinado o blended learning, y se presentaron diferentes Sistemas Virtuales de Aprendizaje como Moodle, Blackboard y Sakai. Entre estos sistemas, durante el curso se expusieron en detalle los conocimientos y herramientas para el uso de Moodle, por tratarse del sistema seleccionado por la Universidad Nacional del Litoral para dar soporte al "Entorno Virtual UNL", ofrecido a su comunidad académica como espacio virtual de apoyo a la educación presencial.

Se debatieron diferentes alternativas y niveles de integración de las TAC, ya sea utilizando la plataforma como repositorio o como 

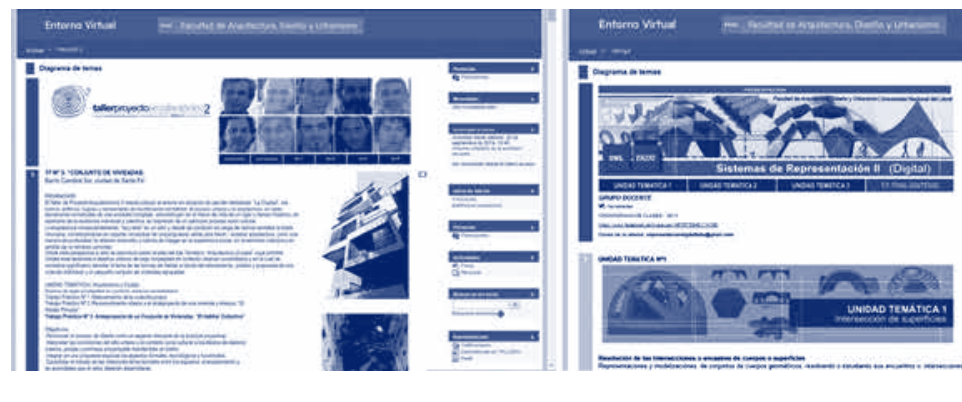
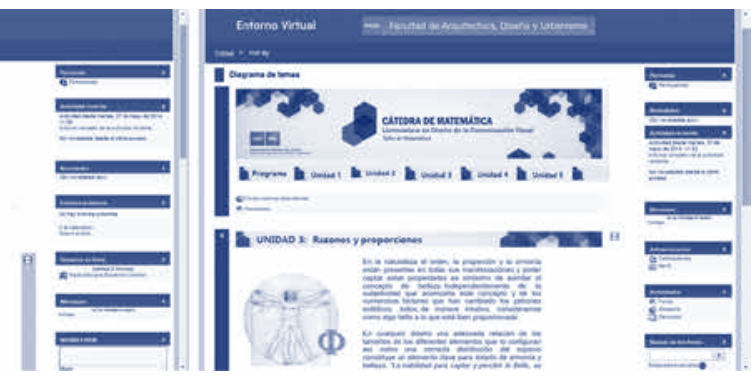

Figura 2: Entornos Virtuales desarrollados en la plataforma educativa Moodle, propuestos como repositorios de material educativo de las siguientes cátedras (de izquierda a derecha): Taller de Proyecto Arquitectónico II (Cátedra Irigoyen), Sistemas de Representación (Cátedra Latorre); y Taller de Matemática de la Carrera de Licenciatura en Diseńo de la Comunicación Visual.

"aula virtual", así como estrategias para entrelazar las actividades entre los espacios físicos y virtuales. A los fines de la producción de los materiales educativos destinados a la plataforma, se desarrollaron los principales softwares de edición y optimización para publicación de textos e imágenes en la web; y de creación y edición de videos y presentaciones dinámicas multimedia.

Respecto de los instrumentos y recursos a incorporar en el aula virtual y sobre la base de la convergencia de medios, se propuso la integración de cursos y recursos de Acceso Abierto de la Web 2.0 con fines educativos: Miríada, MOOC, MIT OpenCourseWare, Stanford Online, etc., así como Facebook, Google+, Twitter, Skype, Hangout, Flickr, Issuu, Youtube, Vimeo, Blogger, Wordpress, Wix, etc.

Se abordaron herramientas de comunicación y de construcción colaborativa de conocimientos, relacionadas con actividades específicas de las disciplinas proyectuales: debates, trabajos prácticos y "enchinchadas" virtuales. Se brindaron instrumentos para la creación y administración de foros, glosarios, wikis y bases de datos así como también, estrategias para incentivar la participación y los aportes colaborativos de los alumnos. A los recursos que ya posee Moodle, se sumaron otros complementarios como Google Drive, DropBox, Mediafire, Conceptboard, etc.

\section{Evaluación}

Como los sistemas de evaluación están íntimamente relacionados con el modo en que se concibe el aprendizaje, para la evaluación del curso se utilizaron métodos colaborativos basados en una concepción abierta y compartida del conocimiento: la evaluación por pares-EP- y la co-evaluación. Hubo dos instancias de evaluación: una parcial, considerada parte importante del proceso, que se diseñó para brindar una retroalimentación prospectiva o pro-alimentación; y la evaluación final que se realizó por el sistema de co-evaluación, correspondiendo un $50 \%$ a la EP y un $50 \%$ a las efectuadas por los docentes a cargo.

El proceso de evaluación se sistematizó a partir del debate entre todos los profesores, con objeto de lograr un acuerdo inicial y aclarar qué elementos integrarían el procedimiento: las tareas a realizar y los productos esperados; las categorías y criterios de evaluación; las personas que intervendrían, los momentos y métodos de evaluación; y la justificación de las valoraciones realizadas.

\section{Resultados}

Como resultado del curso, se desarrollaron 18 propuestas didácticas innovadoras con sus correspondientes aulas virtuales, las cuales integraron una multiplicidad de recursos y servicios de Acceso Abierto.

Para analizar las propuestas realizadas, las mismas se agruparon en tres niveles de desarrollo, tomando como indicador el grado de interacción propuesta, siendo este el factor determinante que posibilita ampliar de manera efectiva los espacios de relación y aprendizaje (Reig, 2013).

Desde esta óptica, es posible identificar un primer grupo o nivel de desarrollo, conformado por las propuestas de 3 asignaturas, donde los entornos virtuales propuestos se utilizan sólo a modo de repositorio de un vasto material educativo elaborado por las cátedras. No se proponen interacciones con los alumnos, ni se incorporan elementos que permitan evaluar la interrelación entre la presencialidad y el entorno virtual, en cuanto a la realización de actividades que vinculen uno y otro ámbito. Las aulas virtuales sólo se proponen como espacios para el acceso y la revisión de contenidos. Sí se destaca en las 3 propuestas, la importante producción de material educativo desarrollado por las cátedras, y la diversidad de recursos utilizados: clases, textos, videos, presentaciones de PowerPoint y Prezi, etc., que han sido organizados en bases de datos en el entorno virtual, posibilitando su acceso a los alumnos (Fig. 2).

El segundo grupo o nivel de desarrollo corresponde a aquellas propuestas desarrolladas también en Moodle, pero que además de sistematizar y habilitar el acceso online a los diversos recursos y materiales educativos, implementan herramientas dirigidas a propiciar la interrelación alumno-alumno y alumno-docente. Dentro de este grupo se pueden considerar 7 propuestas realizadas por las cátedras, que contemplan en sus entornos virtuales prácticas de interacción y de construcción colaborativa de conocimientos a través de foros, glosarios y bases de datos. Proponen también actividades en las que se interrelacionan las instancias presencial y virtual, aprovechando las ventajas de vincular uno y otro ámbito en el aprendizaje de las disciplinas proyectuales (Fig. 3).

Por último, el tercer nivel de desarrollo identificado corresponde a aquellas propuestas que han avanzado además en la integración a sus entornos virtuales en Moodle, de las redes sociales y diversos recursos de la Web 2.0 con fines educativos. Es posible agrupar aquí 8 de las 18 propuestas desarrolladas por las cátedras, que partiendo siempre de la plataforma institucional, han desarrollado espacios interrelacionados y complementarios en cuanto a contenidos y funciones, bajo una identidad gráfica común, incorporando según los casos, espacios en: Facebook, Twitter, Blogs, Pinterest, Youtube, Flickr, Slideshare, etc. 


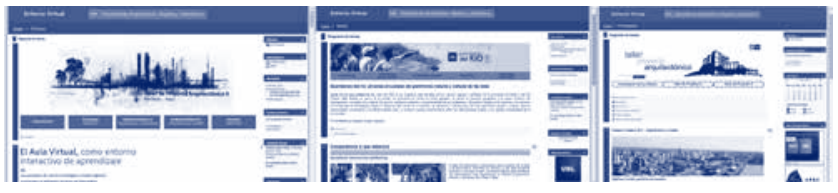

Figura 3: Ejemplos de propuestas desarrolladas en Moodle que incorporan herramientas de interacción y de construcción colaborativa: Taller de Proyecto Arquitectónico II (Cátedra Falco); Proyecto de Extensión de Interés Social: "Guardianes del río. Trabajo sustentable para jóvenes intérpretes del patrimonio de Alto Verde e islas"; y Taller de Proyecto Arquitectónico III y IV (Cátedra Melhem / Irigoyen).

En estas propuestas definitivamente se han repensado las estrategias didácticas, contenidos y actividades, logrando no sólo utilizar el entorno virtual como espacio de acceso a materiales educativos y a la interacción con los alumnos, sino que se ha conseguido ampliar los espacios de aprendizaje y de interrelación, a través de la incorporación de otros recursos disponibles en la web 2.0 (Fig. 4).

\section{Conclusiones}

En principio consideramos importante destacar el efecto multiplicador que ha producido el curso en el desarrollo de aulas virtuales en la FADU-UNL. Como indicador podemos mencionar que si bien en el marco del curso se crearon 18 aulas virtuales de distintas cátedras, al momento de la presentación de esta ponencia ya son 44 las aulas virtuales de esta facultad que se encuentran funcionando en el Entorno Virtual UNL (http://entornovirtual.unl.edu.ar/). Otro indicador que señala su impacto positivo, es un relevamiento realizado entre los estudiantes que cursan actualmente el 1er. ańo de la carrera de Arquitectura y Urbanismo, y de la Licenciatura en Diseño de la Comunicación Visual, los cuales al ser consultados acerca de si utilizan el aula virtual de las asignaturas, respondieron afirmativamente en un $80 \%$.

Podemos concluir que los objetivos planteados para el curso (repensar las estrategias didácticas, contenidos, actividades, trabajos prácticos y criterios de evaluación de las asignaturas de la FADU, integrando las potencialidades de las TAC), se han cumplido ampliamente. Pero el cambio aún está en proceso y estos resultados incipientes no permiten aún evaluar el impacto cualitativo que pueden tener las nuevas propuestas didácticas en la construcción de aprendizajes significativos por parte de los alumnos.

Sí podemos afirmar que la experiencia fue muy enriquecedora para todos los profesores involucrados, ya que permitió conocer una diversidad de temáticas y enfoques; demostró la apertura crítica de los docentes en los debates y el compromiso ético con su rol, que se evidenció en los generosos aportes que realizaron en los foros. La intención es continuar alimentando espacios que posibiliten repensar la tarea docente en forma conjunta y compartir esta dinámica con el resto de las asignaturas de la facultad, para dar un nuevo sentido a las prácticas educativas y motivar a los estudiantes a crear sus entornos personales de aprendizaje, para alcanzar la autogestión de su formación.

En palabras de docentes que participaron del curso:

"Los foros deberían permanecer abiertos para que nosotros también podamos compartir materiales, articular mejor ciertas asignaturas y tener nuestro propio espacio de construcción colectiva."

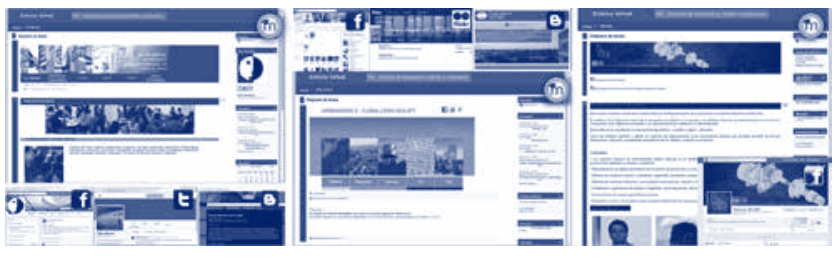

Figura 4: Propuestas desarrolladas en Moodle, que se complementan con espacios creados en redes sociales: Taller de Proyecto Arquitectónico III y IV (Cátedra Mansur) y Urbanismo II (Cátedra Caballero / Soijet) de la Carrera de Arquitectura y Urbanismo; y Sistemas de Representación I y II de la Licenciatura en Diseño Industrial.

"Las TIC y los EVA son instrumentos que nos permiten actualizar la dinámica de enseñanza-aprendizaje. Nos posibilitan redefinir roles y dominar un espacio que cada vez se torna más ubicuo." "... es un esfuerzo grande incorporar las tecnologías, me gustaría tener la destreza de los jóvenes, pero lo estamos intentando..."

\section{Agradecimientos}

Este trabajo es parte del proyecto de investigación CAI+D 2011 N³56 "Diseño, desarrollo y evaluación de espacios-interfaz destinados a actividades de docencia, investigación y extensión en la universidad pública argentina”, subsidiado por la Universidad Nacional del Litoral, Santa Fe, Argentina.

Agradecemos especialmente a los docentes invitados (Arqs. Pieragostini y Stipech) y a los docentes colaboradores, en particular a las arquitectas Cecilia Zorzón y Analía Raffin, y a la LDCV Cecilia Scándolo. Agradecemos también a todos los docentes que han participado del curso en carácter de alumnos, por su interés, aportes y compromiso.

\section{Referencias}

Bessone, M., \& Garramuño, S. (2009). Un nuevo tipo de racionalidad construida a partir de la relación entre sujetos, objetos y multimedios. En 2do Congreso Internacional de Educación. Santa Fe: UNL.

Foro de debate entre docentes del Curso "Interfaces Educativas. Estrategias, Instrumentos y Espacios para Propuestas Didácticas Innovadoras", FADU UNL. Recuperado de http://entornovirtual.unl.edu.ar/course/view.php?id=899

Freire, P. (1989). Alfabetización. Lectura de la palabra y lectura de la realidad. Madrid: Paidós-MEC.

Grane Oró, M. (2013). Relaciones de diseño en entornos de formación online. En La universidad en la nube. Recuperado de http://www.lmi.ub.edu/transmedia21/vol6/La_Universidad_en_la_Nube.pdf

Piscitelli, A. (2009). Nativos digitales. Dieta cognitiva, inteligencia colectiva y arquitecturas de la participación. Buenos Aires: Ediciones Santillana.

Reig, D., \& Vilches, L. (2013). Los jóvenes en la era de la hiperconectividad: tendencias, claves y miradas. Madrid: Fundacion Telefónica.

Universidad Nacional del Litoral. (2010). Plan de Desarrollo Institucional 2010-2019. Líneas de Orientación Principales II: Calidad en enseñanza, investigación y extensión. Santa Fe. 